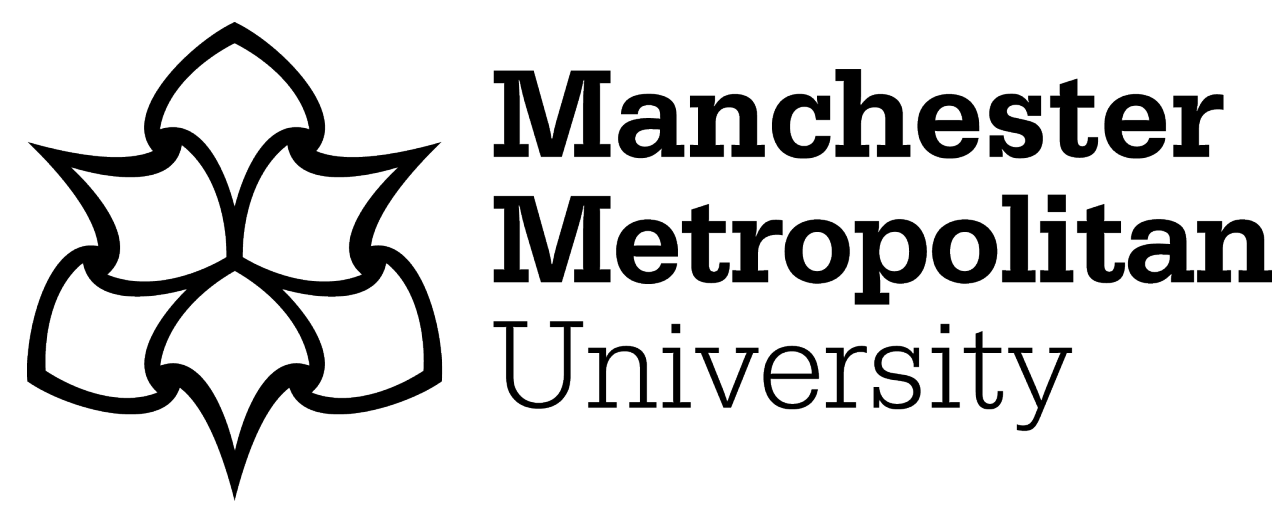

Rukasha, T, Woolley, SI and Collins, T ORCID logoORCID: https://orcid.org/0000-0003-2841-1947 (2020) Wearable epilepsy seizure monitor user interface evaluation: An evaluation of the empatica 'embrace' interface. In: Adjunct - Proceedings of the 2020 ACM International Joint Conference on Pervasive and Ubiquitous Computing and Proceedings of the 2020 ACM International Symposium on Wearable Computers.

Downloaded from: https://e-space.mmu.ac.uk/626524/

Publisher: Association for Computing Machinery

DOI: https://doi.org/10.1145/3410530.3414382

Please cite the published version 


\title{
Wearable Epilepsy Seizure Monitor User Interface Evaluation
}

\author{
An Evaluation of the Empatica 'Embrace' Interface
}

\author{
Tendai Rukasha \\ School of Computing and \\ Mathematics \\ Keele University \\ Staffordshire, UK \\ t.rukasha@keele.ac.uk
}

\author{
Sandra I Woolley \\ School of Computing and \\ Mathematics \\ Keele University \\ Staffordshire, UK \\ s.i.woolley@keele.ac.uk
}

\author{
Tim Collins \\ Department of Engineering \\ Manchester Metropolitan University \\ Manchester, UK \\ t.collins@mmu.ac.uk
}

\begin{abstract}
Wearable health devices have the potential to incentivize individuals in health-promoting behaviors and to assist in the monitoring of health conditions. Wearable epilepsy seizure monitoring devices are now evolving that can support individuals and their caregivers via the automated sensing, reporting and logging of epileptic seizures. This work contributes a novel reflection on the interface requirements of wearer users and non-wearer stakeholder users. We evaluate the "guessability" of the light pattern interface of the Empatica Embrace wrist-worn epileptic seizure monitor and provide box plot results for eight interface indications. We also report summarised feedback from a heuristic analysis with fourteen participant evaluators. The results indicate some satisfaction with the minimal aesthetic of a simple light pattern interface as well as some concerns about confusion between different indications, accessibility and reliance on recall.
\end{abstract}

\section{CCS CONCEPTS}

\section{- Human-centered computing $\rightarrow$ Interaction design.}

\section{KEYWORDS}

Wearable Computing; Interface Evaluation; Health Technology; Epilepsy Monitoring; Heuristic Evaluation; Usability

\section{ACM Reference Format:}

Tendai Rukasha, Sandra I Woolley, and Tim Collins. 2020. Wearable Epilepsy Seizure Monitor User Interface Evaluation : An Evaluation of the Empatica 'Embrace' Interface. In Adjunct Proceedings of the 2020 ACM International foint Conference on Pervasive and Ubiquitous Computing and Proceedings of the 2020 ACM International Symposium on Wearable Computers (UbiComp/ISWC '20 Adjunct), September 12-16, 2020, Virtual Event, Mexico. ACM, New York, NY, USA, 4 pages. https://doi.org/10.1145/3410530.3414382

\section{INTRODUCTION}

Patient monitoring systems capable of accurate recording in the realworld, during the activities of everyday living, create opportunities to make real-time assessments of patient well-being, respond to potentially critical events and support clinical decision making [8].

Permission to make digital or hard copies of part or all of this work for personal or classroom use is granted without fee provided that copies are not made or distributed for profit or commercial advantage and that copies bear this notice and the full citation on the first page. Copyrights for third-party components of this work must be honored. For all other uses, contact the owner/author(s).

UbiComp/ISWC '20 Adjunct, September 12-16, 2020, Virtual Event, Mexico

(C) 2020 Copyright held by the owner/author(s).

ACM ISBN 978-1-4503-8076-8/20/09.

https://doi.org/10.1145/3410530.3414382
Epilepsy is a neurological condition that affects 50 million people worldwide [11]. While antiepileptic drugs can control the seizures of many individuals, more than $30 \%$ of people with epilepsy have drug-resistant seizures [9]. Epileptic seizure types vary considerably between convulsive and non-convulsive seizures including 'tonic' and 'clonic' muscular contractions and relaxations, 'atonic' losses of muscle strength and 'absence' episodes where individuals can lapse awareness and appear detached. For epileptic individuals, the hailing of timely care with automated messages at seizure onset has the potential to reduce injuries and, potentially, save lives.

Epilepsy seizure detection and wearable patient monitoring are active areas of research but there is currently a lack of work evaluating the seizure monitoring technologies currently available to individuals and researchers [7]. This work makes a novel contribution to this area.

\subsection{Wearable Device Interfaces}

Achieving useful and unambiguous information delivery via the small screens and minimal interfaces of wearable devices poses interesting design challenges $[5,13,14]$. At the same time, it is important that devices are aesthetically acceptable [3] and, particularly in the case of health-condition monitoring, it is important that devices are discreet [10] and do not stigmatize wearers [4].

Minimal interface indicators may very quickly become familiar to individuals wearing devices every day. But, in critical healthcare applications there are often other stakeholder users beyond the wearer users and, during critical episodes such as an epileptic seizure, the wearer may be incapacitated or confused for some extended period of time during and after the event.

Examples of non-wearer stakeholder users include a parent or grandparent, teacher, caregiver, colleague, classmate, friend, or First Aid responder. These non-wearer stakeholders may normally have little reason to observe the interface or respond to low priority indications such as "Battery Low". However, the correct identification of a seizure ("Unusual Event Detected") indication could be an important source of seizure corroboration. A correctly interpreted display could also provide some reassurance about automated messaging that could reduce the responder's burden of seizure reporting and messaging. Likewise, the misinterpretation of a non-seizure display as a seizure could have consequences that, like false alarms in general, can disincentivize users.

\subsection{The Empatica Embrace}

The Empatica Embrace epilepsy seizure monitor is one of the few currently available wearable epilepsy seizure monitors [2]. It has a 


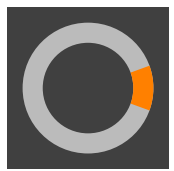

Battery Low

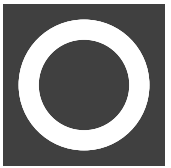

Rebooted
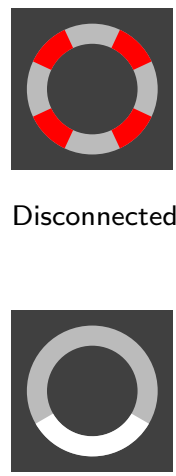

Reconnected
Disconnected

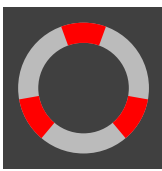

General

Connection Problem

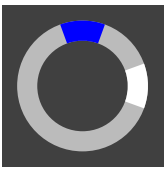

Time

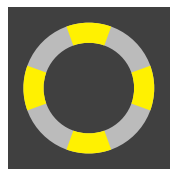

Memory Full

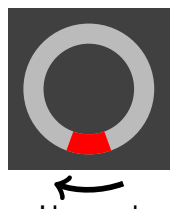

Unusual

Event

Detected
Figure 1: Embrace LED Interface Examples.

multicolor LED (light-emitting diode) interface that includes blinking and rotating animations indicating a range of conditions and states as indicated by the illustrations in Figure 1. Compared to visually-richer displays the LED interface has benefits in terms of aesthetics, internationalization and energy consumption, but has potential drawbacks in terms of usability.

\section{EVALUATION}

\subsection{Method and Materials}

Fourteen Computer Science students and researchers experienced in heuristic evaluation were recruited according to Keele University Faculty of Natural Sciences Research Ethics Committee approval (NS-200058) to evaluate the LED interface of the Empatica Embrace wearable seizure monitor. For repeatability $[1,12]$, the device version was an Empatica Embrace wristband EMB-MB-S (purchased 26th February 2019 with firmware version current between 11th to 13th March 2020).

Participants comprised two academic staff members, three $\mathrm{PhD}$ researchers, and four masters and five undergraduate Computer Science students. Seven participants reported ownership or experience of using wearable health trackers.

Participants were shown each of the eight animated interface indications shown in Figure 1 and were asked to guess on a scale of 5-1 (5 = definitely is and 1 = definitely isn't) what each of eight LED interface patterns signified: Battery Low, Disconnected, General Connection Problem, Memory Full, Rebooted, Reconnected, Time and Unusual Event Detected. The LED patterns were displayed in random order (indicated by Figure 2 labels Q1-Q8). On completion, participants were shown the correct answers for each condition and asked to complete a heuristic evaluation based on Neilsen's 10 Usability Heuristics for User Interface Design [6]: 1) visibility of system status, 2) match between system and the real world, 3) user control and freedom, 4) consistency and standards, 5) error prevention, 6) recognition rather than recall, 7) flexibility and efficiency of use, 8) aesthetic and minimalist design, 9) help users recognize, diagnose, and recover from error, and 10) help and documentation. Participant evaluations were audio recorded and summarized.
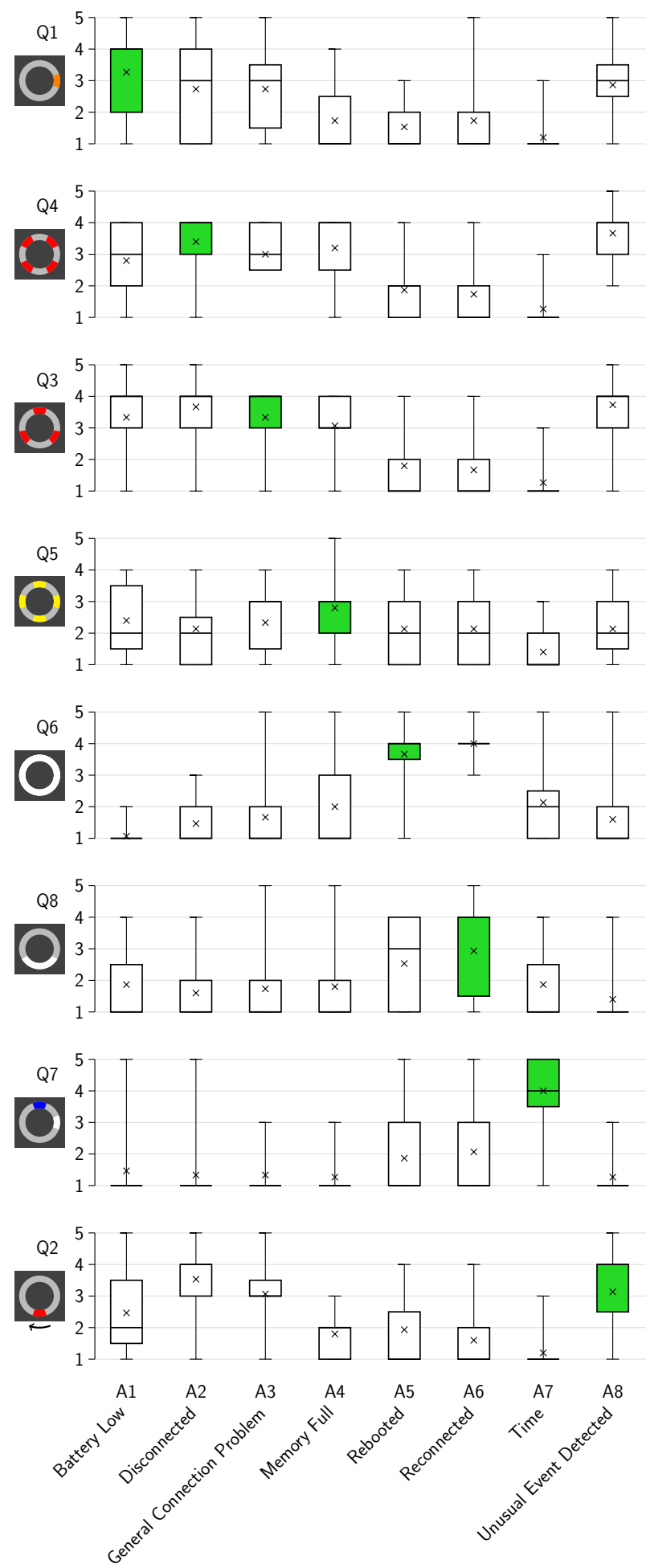

Figure 2: Guessability Box Plots. Participant interface guesses ( 5 = definitely is, 1 = definitely isn't). Correct instances are shaded in green, " $x$ " marks mean, bar marks median and box and whiskers indicate interquartile range and $\max / \mathrm{min}$, respectively. 
Table 1: User Interface Design Heuristics [6] with Summarized Descriptions and Participant Evaluations.

Heuristics

Visibility of system status:

The system keeps users informed of what is going on, through appropriate feedback within reasonable time.

Match between system and the real world: The system should speak the users' language and follow real-world conventions in a natural and logical order.

\section{User control and freedom:}

Support undo and redo and have an "emergency exit”.

\section{Consistency and standards:}

Users should not have to wonder about meanings (device should follow conventions).

\section{Error prevention:}

A design that avoids errors and requests user confirmations.

\section{Recognition rather than recall:}

Users should not have to remember information from one part of the dialog to another.

\section{Flexibility and efficiency of use:}

The system should be able to efficiently cater for both inexperienced and experienced users.

\section{Aesthetic and minimalist design:}

Dialogues should not contain information which is irrelevant.

Help users recognize, diagnose, and recover from errors:

Error messages should specify the problem and suggest a solution.

\section{Help and documentation:}

The system should provide help and documentation (easy to search, focused on the user's task, list steps to be carried out, and should not be too large).

\section{Participant Evaluations}

Some evaluators reported the LEDs as visible and clear but most identified ambiguities.

"About half the LEDs made sense."

"Once the user knows the patterns it could be readable."

"To the unversed person it seems confusing..."

Several evaluators reported a good match for the red color and a warning condition. Opinions varied about the use of white and orange LEDs. Time interface was thought to be intuitive. There was uncertainty about the animations.

"The system does not speak our language or use conventional symbols/signs"

"Red indicates a serious problem."

"Some animations matched real world... most do not."

Most participants felt that this heuristic was not applicable but one evaluator suggested customization control.

Evaluators generally agreed on the internal consistency of the LED displays but did not agree on a consistent standard beyond the use of red for warning. "LEDs don't seem consistent with other products I am aware of."

Most evaluators agreed that, although it is clear when an error or problem has occurred, it was not clear what the error condition was.

"Where the LED shows red, this is most obvious that there is an issue, but difficult to discern what the error it is."

There were also concerns about the accessibility of the display for color blind individuals.

Although there were some intuitive elements of the interface, most evaluators felt the interface relied largely on recall.

"The problem is having to remember what it means..."

"You would have to rely on memorizing the LED patterns..."

Evaluators agreed that the interface was efficient and international.

Some evaluators liked the minimalist aesthetic, but most felt it was too minimalistic.

"Possibly too minimalistic with such a variety of meanings..."

"A lack of text may make it hard to remember the meanings..."

Evaluators expressed different opinions but generally agreed that displays were recognizable if LED patterns were learned, but no indications were given about recovery.

"If users know the meanings, displays are distinct."

"There is little help provided for the user, if they don't know what the lights mean, they won't know what to do."

Participants agreed that there was no help available via the interface.

"None is provided on the interface leading to a reliance on recall or reference to a manual." 


\section{RESULTS}

Figure 2 presents box plot results for the 5-1 $(5=$ definitely is and $1=$ definitely isn't) Likert scale guesses for each of the eight Embrace LED interface examples illustrated. Ideally, the correct LED patterns (shaded in green) would have averages close to 5 and the all incorrect conditions would have averages close to 1 .

Table 1 summarizes the participant evaluations for each of the 10 Nielsen user interface design heuristics [6].

\section{DISCUSSION}

As demonstrated in Figure 2 by the quantity of average guess values between 2 and 4, as well as the similarity of scores between some interface displays, participants found it difficult to disambiguate between sets of conditions. For example, participants could not discern between the orange and red Battery Low, Disconnected and General Connection Problem light patterns: all three received averages of 2.5 to 3.5 ( 3 = unsure) no matter which pattern was displayed. Similarly, the white Rebooted and Reconnected LED patterns were confused with each other.

The Time display was the most recognized display. Only one participant was confident the Time display was not Time and, at most, one participant guessed that Battery Low, Disconnected and General Connection Problem, were Time indicators.

Unfortunately, the spinning red Unusual Event Detected display that can signify a seizure was not guessed well and was confused with Battery Low, Disconnected and General Connection Problem. When displayed, to participants the Unusual Event Detected display received an average score for the correct answer of 3.13 ( $3=$ unsure) which was lower than the (incorrect) Disconnected guess that received an average of 3.53. Overall, for four out of the eight displays, at least one incorrect answer had a higher average guess score than the correct answer.

In Table 1, the heuristic feedback summarises the opinions amongst participant evaluators that, on the one hand, recognize the simplicity, clarity and potential memorability of the display and, on the other, raises concerns about the reliance on recall and the potential for confusion. For example, one evaluator observed that the interface was "Quite aesthetically pleasing but as intuitive as a Star Trek control panel". The use of color, e.g., "Red indicates a serious problem" was seen as appropriate as a real-world convention but some concerns were raised about accessibility for individuals with color-vision deficiencies.

\section{CONCLUSIONS}

Minimal light pattern displays have a pleasing aesthetic but can be confusing to users lacking familiarity with the interface. Ideally, each displayed pattern could be correctly guessed from the set of possible meanings.

There is need for further research and improvements in the design of interface displays for wearable devices and particularly for devices used in critical health monitoring scenarios with wearer users and non-wearer user stakeholders.

\section{ACKNOWLEDGMENTS}

Tendai Rukasha thanks Evaresto Rukasha and Betty Rukasha for funding the $\mathrm{PhD}$ research.

\section{REFERENCES}

[1] Tim Collins, Sandra I. Woolley, Salome Oniani, Ivan Miguel Pires, Nuno M. Garcia, Sean J. Ledger, and Anand Pandyan. 2019. Version Reporting and Assessment Approaches for New and Updated Activity and Heart Rate Monitors. Sensors 19, 7 (Apr 2019), 1705. https://doi.org/10.3390/s19071705

[2] Empatica Inc. Boston, USA and Empatica Srl, Milano, Italy. 2020. Empatica Embrace. Available online: https://www.empatica.com (accessed on 12 May 2020).

[3] Jutta Fortmann, Heiko Müller, Susanne Boll, and Wilko Heuten. 2013. Illumee: aesthetic light bracelet as a wearable information display for everyday life. In Proceedings of the 2013 ACM conference on Pervasive and ubiquitous computing adjunct publication. ACM Press, 393-396. https://doi.org/10.1145/2494091.2495970

[4] Dongni Johansson, Kristina Malmgren, and Margit Alt Murphy. 2018. Wearable sensors for clinical applications in epilepsy, Parkinson's disease, and stroke: a mixed-methods systematic review. Fournal of Neurology 265, 8 (Feb 2018), 1740-1752. https://doi.org/10.1007/s00415-018-8786-y

[5] Vivian Genaro Motti and Kelly Caine. 2016. Smart Wearables or Dumb Wearables? Understanding how Context Impacts the UX in Wrist Worn Interaction. In Proceedings of the 34th ACM International Conference on the Design of Communication. ACM Press, 1-10. https://doi.org/10.1145/2987592.2987606

[6] Jakob Nielsen. 1994. Enhancing the explanatory power of usability heuristics. In Proceedings of the SIGCHI conference on Human Factors in Computing Systems. ACM Press, 152-158. https://doi.org/10.1145/191666.191729

[7] Tendai Rukasha, Sandra I Woolley, Theocharis Kyriacou, and Tim Collins. 2020. Evaluation of Wearable Electronics for Epilepsy: A Systematic Review. Electronics 9, 6 (Jun 2020), 968. https://doi.org/10.3390/electronics 9060968

[8] David Infante Sanchez, Sandra I. Woolley, Tim Collins, P. Pemberton, T. Veenith, D. Hume, K. Laver, and C. Small. 2017. The Quantified Outpatient - Challenges and Opportunities in $24 \mathrm{hr}$ Patient Monitoring. In Informatics for Health, Manchester. Medical Informatics Europe. https://doi.org/10.13140/RG.2.2.33571.86562

[9] Jiyao Sheng, Shui Liu, Hanjiao Qin, Bingjin Li, and Xuewen Zhang. 2018. DrugResistant Epilepsy and Surgery. Current Neuropharmacology 16, 1 (Dec 2018), 17-28. https://doi.org/10.2174/1570159x15666170504123316

[10] Sara Katherine Simblett, Andrea Biondi, Elisa Bruno, Dominic Ballard, Amanda Stoneman, Simon Lees, Mark P. Richardson, and Til Wykes. 2020. Patients' experience of wearing multimodal sensor devices intended to detect epileptic seizures: A qualitative analysis. Epilepsy \& Behavior 102 (Jan 2020), 106717. https://doi.org/10.1016/j.yebeh.2019.106717

[11] WHO. 2019. Epilepsy: A Public Health Imperative. World Health Organization: Geneva, Switzerland.

[12] Sandra I. Woolley, Tim Collins, James Mitchell, and David Fredericks. 2019. Investigation of wearable health tracker version updates. BM7 Health \& Care Informatics 26, 1 (Oct 2019), e100083. https://doi.org/10.1136/bmjhci-2019-100083

[13] Cheng Xu and Kent Lyons. 2015. Shimmering smartwatches: Exploring the smartwatch design space. In Proceedings of the Ninth International Conference on Tangible, Embedded, and Embodied Interaction. ACM Press, 69-76. https: //doi.org/10.1145/2677199.2680599

[14] Cheng Zhang, Junrui Yang, Caleb Southern, Thad E Starner, and Gregory D Abowd. 2016. WatchOut: extending interactions on a smartwatch with inertial sensing. In Proceedings of the 2016 ACM International Symposium on Wearable Computers. ACM Press, 136-143. https://doi.org/10.1145/2971763.2971775 